\title{
Consistency, competitive exclusion and coexistence in complex plankton ecosystem models
}

\author{
Cropp, R.A. ${ }^{1} \&$ J. Norbury ${ }^{2}$ \\ ${ }^{1}$ Atmospheric Environment Research Centre, Griffith University, Nathan, Queensland, 4111, Australia \\ ${ }^{2}$ Mathematical Institute, University of Oxford, 24-29 St. Giles, Oxford OX1 3LB, UK
}

\begin{abstract}
We synthesise the generic properties of ecologically realistic multi-trophic level models and define criteria for ecological realism. We define three simple properties that all sensible ecosystem models should have:
\end{abstract}

1. Independence of scale: the functions that describe the change in the size of the population $\left(x_{i}\right)$ are independent of the scale at which we consider the population, that is:

$$
\frac{1}{x_{i}} \frac{d x_{i}}{d t}=f_{i}\left(x_{1}, x_{2}, \cdots, x_{n} ; N\right) \text { for } i=1,2, \cdots, n .
$$

2. Conservation of mass: we assume that mass of a key limiting nutrient $(N)$ is conserved, with implicit remineralisation of detritus into inorganic nutrient slaved to the ecosystem, that is:

$$
x_{1}+x_{2}+\square+x_{n}+N=N_{T} \Leftrightarrow \dot{x}_{1}+\dot{x}_{2}+\square+\dot{x}_{n}=-\dot{N} .
$$

We scale the system so that the total nutrient $N_{T}=1$, with $0 \leq x_{i} \leq 1$ and $0<x_{1}+x_{2}+\square+x_{n}<1$ and use equation (2) to eliminate $N$ from equation (1). The lowest trophic level ( $x_{1}$ at least) therefore grows on inorganic nutrient and must be an autotroph.

3. Resource limitation: we stipulate that every population must be explicitly limited by a finite resource $\left(R_{i}\right)$, that is, the rate at which the population can grow decreases as availability of its limiting resource decreases. This may be expressed formally by the resource ray gradient condition:

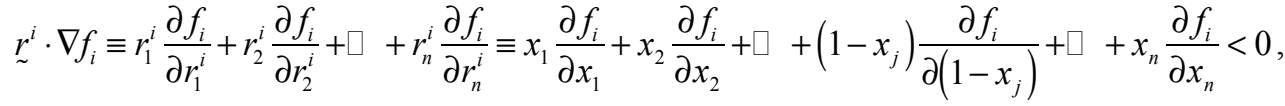

where ${\underset{\sim}{r}}^{i}=\left(x_{1}, \cdots, 1-x_{j}, \cdots, x_{n}\right)$ is a ray spanning the resource space for species $x_{i}$ that feeds on species $x_{j}$. To be a sensible ecology, the ray sign conditions $f_{i}\left(R_{i}=1\right)>0>f_{i}\left(R_{i}=0\right)$ where $R_{i}$ is the amount of resource $i$ must also be met.

This defines an "ecospace" in which all ecologically realistic dynamics are confined, and construct "resource rays" that define the resources available to each species at every point in the ecospace. Resource rays for a species are lines from a vertex of maximum resource to the opposite boundary where no resources are available. The growth functions of all biota normally decrease along their resource rays, and change sign from positive to negative. This property prescribes that each species must have a zero isosurface that divides the ecospace, and provides a simple test for ecological consistency. We use the properties of our consistent ecologies to develop heuristics that illuminate the key mechanisms that allow the coexistence of explicit competitors in these systems. Our approach unifies many theoretical and applied models in a common biogeochemical framework, providing a useful tool to generate new insights into the properties of complex ecosystems.

\section{Keywords}

Conceptual framework, resource space, plankton, consistent ecology, conservation of mass. 


\section{Introduction}

Complex marine plankton models that explicitly resolve the interactions between several functional types or trophic levels are being developed for applications in climate prediction (i.e. PlankTOM10 (Le Quéré et al., 2005)) and fisheries management (i.e. NEMURO (Kishi et al., 2007)). At least one limiting nutrient and three trophic levels or plankton functional types are typically included in such models, and they tend to approximately conserve the quantity of these nutrients, and hence run in an "approximately closed" manner.

We present a simple way to create ecosystem models that are both ecologically realistic and internally or "ecologically consistent" in that all species obey the same basic rules, regardless of their trophic position or interactions. We formulate a 'limiting resource' criterion for each trophic level to prey on inorganic nutrient or other trophic levels. We demonstrate the efficacy of this approach for single resources in simple models, but note that the concept is also central to complex ecologies. This allows a straightforward and ecologically consistent procedure for adding trophic levels to models. This limiting resource approach reveals that the lack of an explicit limiting nutrient is a fundamental inconsistency in many population models.

The principle of competitive exclusion (Gause, 1934; Hardin, 1960) states that the number of species in an ecosystem cannot exceed the number of resources. The apparent exception to this principle obvious in plankton ecosystems led Hutchinson to pose the 'paradox of the plankton' (Hutchinson, 1961). While perhaps no longer paradoxical, in theoretical and applied plankton ecosystem models competitive exclusion remains the dominant outcome of explicit competition for resources (Tilman, 2007; Cropp and Norbury, 2009). These models have contributed little to understanding of competition. The mechanisms that allow explicit competitors to coexist on limited resources in the constant, homogeneous environments that often occur the upper ocean hence remain a mystery (Tilman, 2007). It is on such scenarios that we focus this work, and hence do not consider the influence of spatial heterogeneity that may also support coexistence.

We use the properties of our consistent ecosystems to develop a unifying framework that allows the factors controlling competition and coexistence in theoretical and applied ecosystem models to be explicitly articulated regardless of model complexity. We derive analytic expressions that describe the potential for species to successfully compete and show these are easily obtained for even the most complex ecosystem model. These expressions exactly describe the 'long-term low-density growth rates' central to invasion theory (Chesson, 2000) and may provide general insights into competition properties of many ecosystems. Our framework can accommodate single resource ecosystem models of any complexity, although the analysis of complex models may be tortuous depending on the structure of the equations. There appears to be no intrinsic reason that this framework could not also accommodate models with multiple resources and resource switching, and these will be addressed in future work.

\section{Attributes of Ecologically Consistent Models}

\section{Ecospace}

We measure the populations of the plankton species or functional types by $x_{i}$, the amount of key, or limiting, nutrient $(N)$ that is contained in population $i$. The closed intervals $0 \leq x_{i} \leq N_{T}$ form an ecological space $(E)$ that contains all the ecologically realistic behaviour of the system. Conservation of the total mass of nutrient $\left(N_{T}\right)$ means that $x_{1}+x_{2}+\cdots+x_{n}=N_{T}-N$, and implies a condition on the $f_{i}$ that $x_{1} f_{1}+x_{2} f_{2}+\cdots+x_{n} f_{n}=-d N / d t$. We scale the populations in terms of the fraction of the total amount of nutrient that each population represents (i.e. $\hat{x}_{i}=x_{i} / N_{T}$ and $\hat{N}=N / N_{T}$ ), defining a non-dimensional ecospace $\hat{x}_{1}+\hat{x}_{2}+\cdots+\hat{x}_{n}=1-\hat{N}$. We use this to eliminate $N$ from equation (1), turning the population biology model, in which some populations sizes are unbounded, into an ecology in which all population sizes are limited by finite resources. We drop the hats and define the ecospace:

$$
E \equiv\left\{0<x_{1}+x_{2}+\square+x_{n}<1 ; 0<x_{i} \forall i\right\} .
$$

The conservation of mass condition forms an "ecological lid" at $N=0$; where our requirement that $d N / d t>0$ on $N=0$ (we call this the "rigid lid" condition) constrains the dynamics of the system to lie below the lid. The lid is shown in Fig 1 as the dashed triangular face. 


\section{Resource Space}

The ecospace $E$ defines a resource space of inorganic nutrient for the autotrophs identical to the ecospace, and also resource spaces for heterotroph species that consume other species. These latter resource spaces occupy the same space as the ecospace but differ in that they view the ecospace $E$ from the perspective of that heterotroph (see Fig 1). An $n$ trophic level model will have $n$ explicit resource spaces defined by the model, which we label $E^{1}$ through to $E^{n}$. The resource space $E^{i}$ of species $x_{i}$ has its origin at the point in the ecospace where its resource is maximum, that is, if $x_{i}$ feeds upon $x_{j}$, then its resource space is defined by:

$$
E^{i}=\left\{r_{1}^{i}, r_{2}^{i}, \cdots, r_{n}^{i}\right\}=\left\{x_{1}, \cdots, 1-x_{j}, \cdots, x_{n}\right\} \text { for } 1 \leq i \leq n, i \neq j
$$

Examples of resource spaces are shown in Figures 1 and 2, where in each case we have assumed the simplest case that $x_{i}$ feeds upon $x_{i-1}$. We note that $x_{0}$ is not defined when we consider the resource space for $x_{1}$. In this case the $1-x_{i-1}$ term is not relevant and the resource space for $x_{1}\left(E^{1}\right)$ is just the ecospace $E$. In general, for consistent ecologies, the species occupying the lowest trophic level $\left(x_{1}\right)$ must be an autotroph that feeds only on inorganic nutrient $N$.

\section{Resource Rays}

We define resource rays that may be drawn through, and span, a resource space $\left(E^{i}\right)$. The rays emanate from the origin of $E^{i}$ (which is the relevant pure resource vertex of $E$ where the resource $R^{i}=x_{j}$ is maximal) to the opposite face where the relevant resource level $R^{i}=x_{j}=0$ (Figures 1 and 2). The biological interpretation of the rays is that available resources (inorganic nutrient in the case of autotrophs, but organisms occupying other trophic level in the cases of grazers and carnivores) reduce as one moves from the origin of the ray to the other side.

\section{Ray Gradient Condition}

At each point on a resource ray we describe the $x_{i}$ population's ability to thrive on the quantity of its resource available there by its life function $f_{i}$. Each life function must decrease along its resource rays and the directional derivative of a life function along its resource rays must therefore be negative:

$$
\underset{\sim}{r^{i}} \cdot \nabla f_{i} \equiv r_{1}^{i} \frac{\partial f_{i}}{\partial r_{1}^{i}}+r_{2}^{i} \frac{\partial f_{i}}{\partial r_{2}^{i}}+\square+r_{n}^{i} \frac{\partial f_{i}}{\partial r_{n}^{i}} \equiv x_{1} \frac{\partial f_{i}}{\partial x_{1}}+x_{2} \frac{\partial f_{i}}{\partial x_{2}}+\square+\left(1-x_{j}\right) \frac{\partial f_{i}}{\partial\left(1-x_{j}\right)}+\square+x_{n} \frac{\partial f_{i}}{\partial x_{n}}<0 .
$$

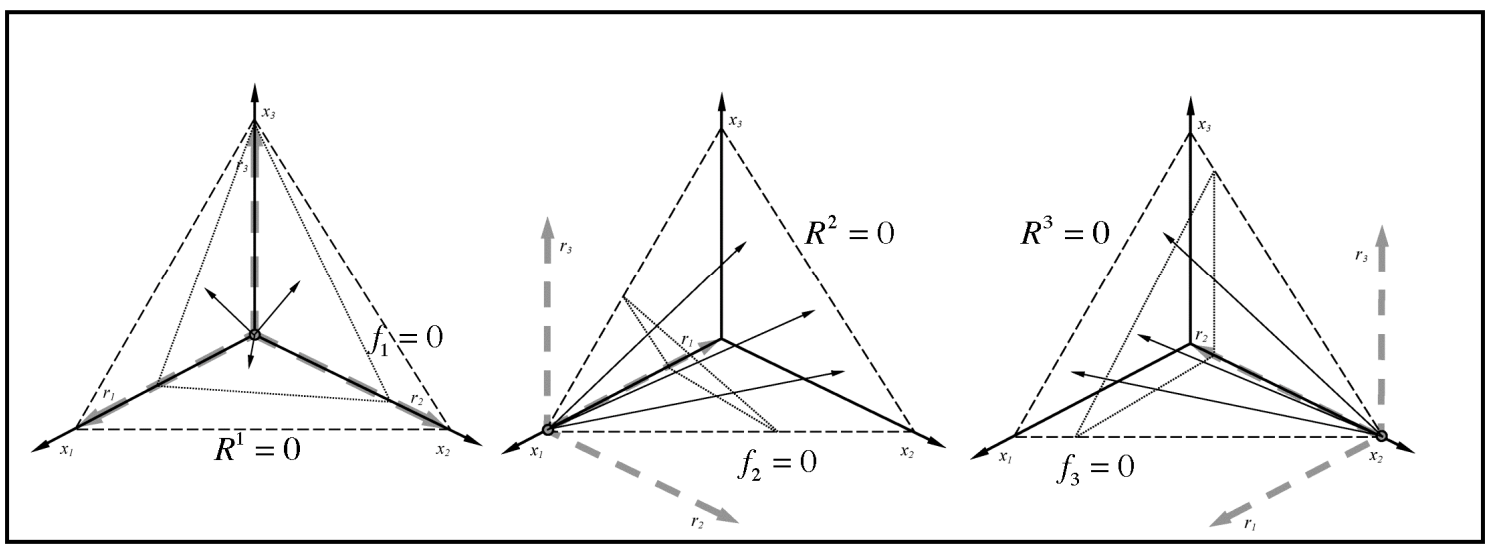

Figure 1. Resource space, rays, ray gradient and ray sign conditions and zero isosurfaces for a three trophic level system. Solid dark arrows are the axes of the ecospace $(E)$, broad dashed grey arrows are the axes of the appropriate resource space $\left(E^{i}\right)$. Dashed lines are the $R^{1}=0$ "lid" imposed on resource $E^{1}$ by the conservation of mass criterion. Dotted lines denote the zero isosurfaces. 


\section{Resource Ray Sign Condition}

A realistic ecosystem model must have the value of each life function $f_{i}$ positive at its maximum resource vertex and negative at its zero resource face, that is:

$$
f_{i}\left(R_{i}=1\right)>0>f_{i}\left(R_{i}=0\right) .
$$

The positive condition constrains the magnitude of the natural mortality rate of each species and the negative condition means that in the absence of resources the species population cannot survive. The key ecological point of this condition is that each life function $f_{i}$ must be negative everywhere on its zero resource face, otherwise the model claims that the species can grow even though it has no resources to support its growth.

\section{Zero Isosurfaces}

Along every resource ray $\underset{\sim}{r^{i}}$ in its resource space $E^{i}$ there exists a point ${\underset{\sim}{*}}_{*}^{i}=\lambda_{*}^{i}\left(r_{1}^{i}, \cdots, r_{n}^{i}\right)$, with $0<\lambda_{*}^{i}<1$, where the growth and loss terms of the population $x_{i}$ balance (i.e. $f_{i}\left(r_{*}^{i}\right)=0=d x_{i} / d t$; note $\lambda_{*}^{i}$ varies as we vary the ray). These points $r_{*}^{i}$ then form the $f_{i}$ zero isosurface (i.e. $f_{i}=0$ in $E$ ). The key attribute of an isosurface is that it must divide the ecospace $E$ into two parts; one part where $x_{i}(t)$ is increasing in $t$, and one where $x_{i}(t)$ is decreasing in $t$.

\section{Critical Points and Stability}

Critical points $\left\{x_{i}^{*}\right\}$ in ecosystem models are points where the state of the system does not change in time (i.e. $\dot{x}_{i}=0$ for all $i$ ). A consistent ecosystem model with $n$ species has $2^{n}$ sets of $n$ equations that describe its critical points $\left\{x_{i}^{*}\right\}, i=1,2, \cdots, n$, that could exist in the $n$-dimension ecological state space. All $n$-species ecosystems have $2^{n}-1$ extinction critical points $(e C P \mathrm{~s})$ where one or more species are extinct (i.e. $\left\{x_{i}^{*}=0\right\}$ for some $i$ ) including the origin of the state space, defined by $\left\{x_{i}^{*}=0\right\}$ for all $i$ where all species are extinct. They also have one coexistence critical point, defined by $\left\{x_{i}^{*} \neq 0\right\}$ for all $i$, where all species coexist, although this does not necessarily reside within the ecospace. The Lyapunov (local) stabilities of the critical points are central to the mechanisms of coexistence.

\section{Extinction in ecosystem models}

Extinction in mathematical models of ecosystems occurs if the model has a stable critical point at which a variable $x_{i}$ representing a species equals zero. Generally, analytic expressions for the eigenvalues $\left(\lambda_{i}\right)$ of the Jacobian matrix of a system, that determine the Lyapunov stability of critical points, are available only for very simple ecosystem models. However, consistent ecosystem models have the property that the eigenvalue $\lambda_{j}$ associated with a species $x_{j}$ at an $e C P$ where $x_{j}^{*}=0$ is given by the value of its life function evaluated at the critical point, that is $\lambda_{j}=\left.f_{j}^{*}\right|_{e C P}$. These 'competition eigenvalues' determine the potential for a species to out-compete established species and grow from very low population levels, and this is the key attribute that makes consistent ecosystem models ideal for investigating competition in complex ecosystems.

\section{Properties of Consistent Ecosystem Models}

\section{Ecological Consistency}

We demonstrate the properties of consistent ecosystem models by considering the model of Hastings and Powell (1991):

$$
\frac{d x}{d t}=x\left(1-x-\frac{a_{1} y}{1+b_{1} x}\right)
$$




$$
\begin{gathered}
\frac{d y}{d t}=y\left(\frac{a_{1} x}{1+b_{1} x}-\frac{a_{2} z}{1+b_{2} y}-d_{1}\right), \\
\frac{d z}{d t}=z\left(\frac{a_{2} y}{1+b_{2} y}-d_{2}\right) .
\end{gathered}
$$

This model is inconsistent because $f_{x}$ (equation (8)) fails the resource ray sign test. This is evident in Figure 2 (left panel) at the top of the ecospace where $f_{x}$ does not form a closed space about the point of maximum resource. The model may be brought into a consistent form by imposing explicit limitation of resource on $f_{x}$ by replacing the carrying capacity in equation (8) with a Michaelis-Menten nutrient uptake term:

$$
\frac{d x}{d t}=x\left(\frac{N}{N+\varepsilon}-x-\frac{a_{1} y}{1+b_{1} x}\right),
$$

where $N=1-x-y-z$ and $\varepsilon$ is the nutrient concentration at which $x$ achieves half its maximum nutrient uptake rate. For $\varepsilon=0$ the revised model is identical to the original population model, but for $\varepsilon>0$ it becomes a consistent ecology. This is evident in Figure 2 (right panel) as the "closing off" of the $f_{x}$ zero isosurface at the top of the ecospace. The chaotic dynamics of the Hastings and Powell model are retained for very small $\mathcal{E}$, but rapidly disappear as $\mathcal{E}$ is increased to realistic levels, suggesting that they only appear in unrealistic parameterisations.
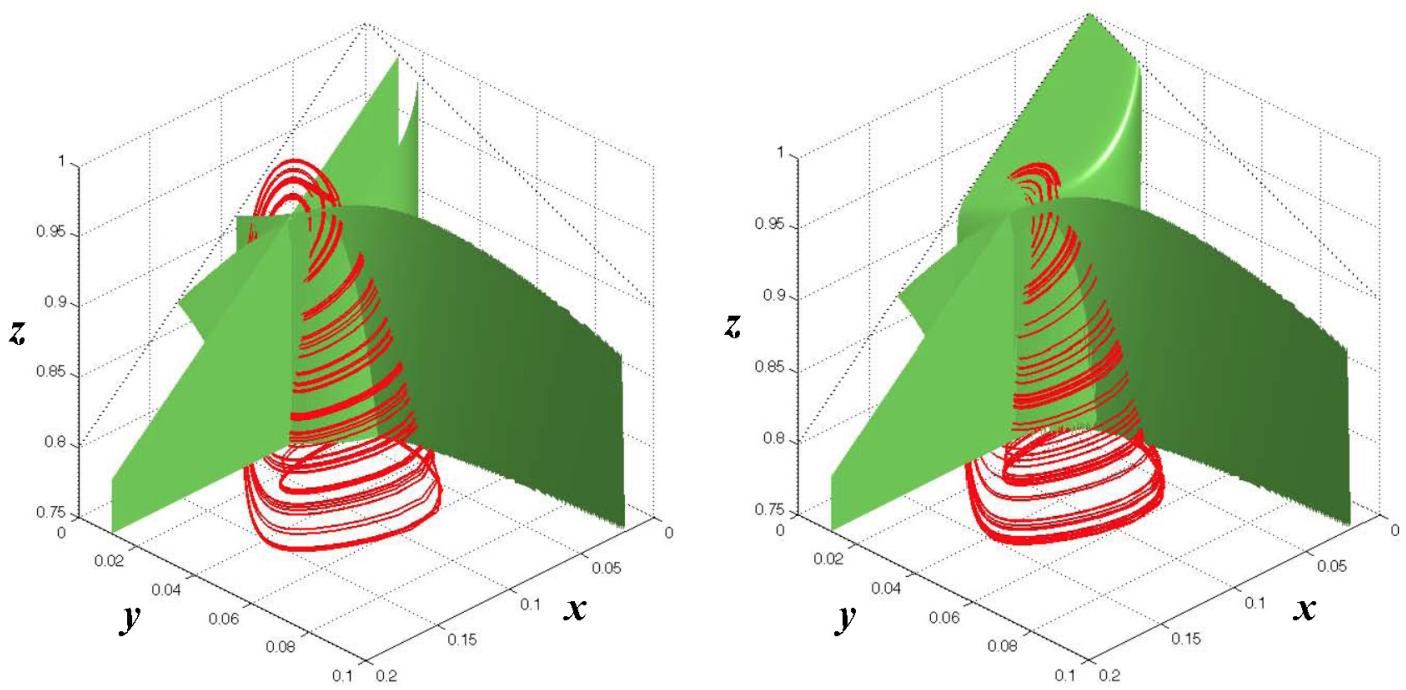

Figure 2. Ecospace, strange attractor and zero isosurfaces for the Hastings and Powell (1991) system (left panel) and its ecologically-consistent equivalent (right panel).

\section{Conditions for coexistence in consistent ecosystem models}

We identify two fundamental ways in which models may have the property that all species remain extant. Ecosystem models may have the structural property that all competition eigenvalues are positive at every $e C P$, or they may be parameterized so that at least one competition eigenvalue is positive at every $e C P$. We shall refer to these as structural coexistence (SC) and parameterized coexistence (PC) systems respectively.

The structural properties of SC systems are ensured if every species has density-independent growth processes coupled with density-dependent loss processes, where we define density-dependence to mean that the process can be written as the product of a function and the species variable $x_{i}^{\omega}$ where $\omega>1$. PC systems are composed of species that have at least one density-independent loss term, for example Holling type I or II grazing or linear mortality. They have the potential for much more varied properties than SC systems, but they are more difficult to construct, as they require the locations of all boundary and interior critical points in the ecological state space to be known. 
Example SC and PC systems

We define four examples each of SC and PC systems of four autotrophs, three autotrophs and a grazer, two autotrophs and two grazers, and one autotroph and three grazers. The generic equations:

$$
\begin{gathered}
\frac{d A_{i}}{d t}=\left(\frac{\mu_{i} N}{N+\kappa_{i}}+\sum_{h=1}^{i-1} \frac{\hat{\varphi}_{h i}\left(1-\psi_{h i}\right) A_{h}^{2}}{A_{h}^{2}+\hat{\kappa}_{h i}}-\sum_{j=i+1}^{x} \frac{\hat{\varphi}_{i j} A_{i} A_{j}}{A_{i}^{2}+\hat{\kappa}_{i j}}-\sum_{k=1}^{y} \frac{\varphi_{i k} A_{i} G_{k}}{A_{i}^{2}+\kappa_{i k}}-\sigma_{i} A_{i}\right) A_{i}, \\
\frac{d G_{k}}{d t}=\left(\sum_{i=1}^{x} \frac{\varphi_{i k}\left(1-\psi_{i k}\right) A_{i}^{2}}{A_{i}^{2}+\kappa_{i k}}+\sum_{p=1}^{k-1} \frac{\tilde{\varphi}_{p k}\left(1-\tilde{\psi}_{p k}\right) G_{p}^{2}}{G_{p}^{2}+\tilde{\kappa}_{p k}}-\sum_{q=k+1}^{y} \frac{\tilde{\varphi}_{k q} G_{k} G_{q}}{G_{k}^{2}+\tilde{\kappa}_{k q}}-\tilde{\sigma}_{k} G_{k}\right) G_{k},
\end{gathered}
$$

are used for the SC systems, where $A$ represents the autotrophs and $G$ denotes the grazers (mixotrophy and omnivory are allowed), and $N$ represents inorganic nutrient where $N=1-\sum A-\sum G$. These equations, and the $P C$ systems described in equations (14) and (15) below are completely described in Cropp and Norbury (in press).
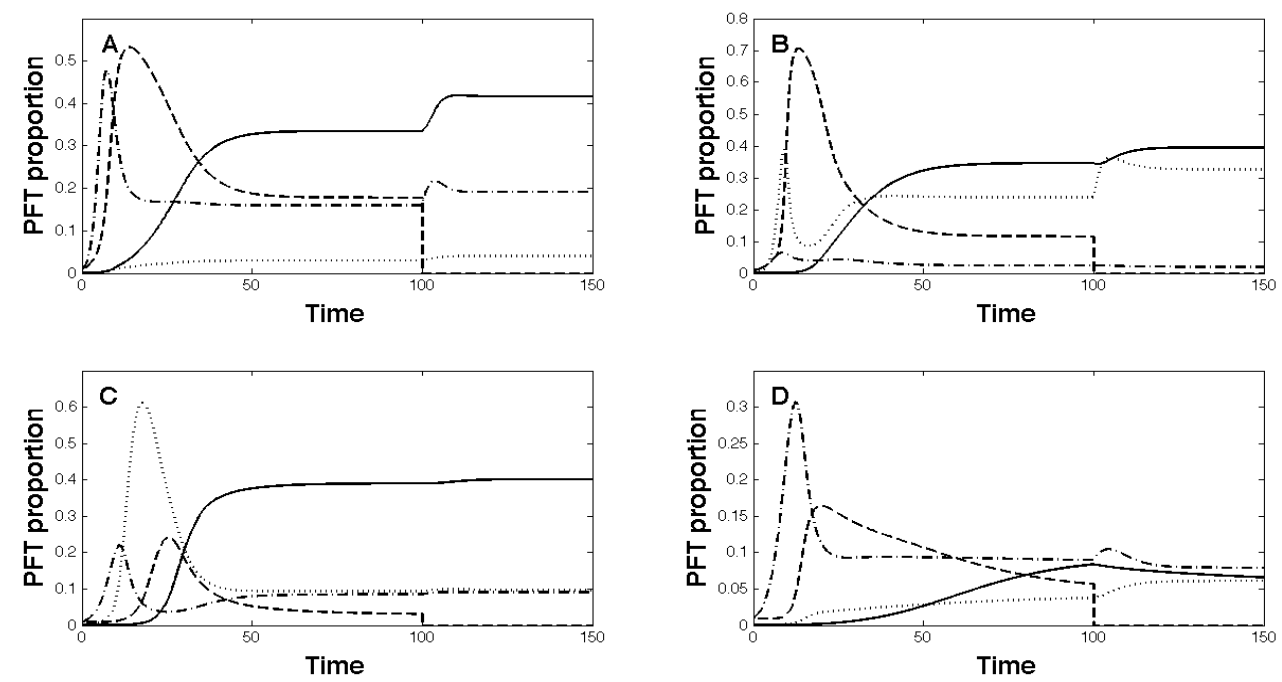

Figure 3. Time series for four example SC ecosystem models: four autotrophs (A); three autotrophs and grazer (B); two autotrophs and two grazers (C); and one autotroph and three grazers (D). The

SC systems have equilibrium dynamics, but may have any dynamics. Populations are shown as proportions of the total functional types (PFTs). Extinction is externally imposed on the third species (dashed line) at time 100 in each simulation. No cascades of extinction occur.

The generic equations:

$$
\begin{gathered}
\frac{d A_{i}}{d t}=\left(\frac{\mu_{i} N}{N+\kappa_{i}}+\sum_{h=1}^{i-1} \hat{\varphi}_{h i}\left(1-\hat{\psi}_{h i}\right) A_{h}-\sum_{j=i+1}^{x} \hat{\varphi}_{i j} A_{j}-\sum_{k=1}^{y} \varphi_{i k} G_{k}-\sigma_{i}\right) A_{i}, \\
\frac{d G_{k}}{d t}=\left(\sum_{i=1}^{x} \varphi_{i k}\left(1-\psi_{i k}\right) A_{i}+\sum_{p=1}^{k-1} \tilde{\varphi}_{p k}\left(1-\tilde{\psi}_{p k}\right) G_{p}-\sum_{q=k+1}^{y} \tilde{\varphi}_{k q} G_{q}-\tilde{\sigma}_{k}\right) G_{k},
\end{gathered}
$$

are used for the PC systems, where in both models $x$ is the number of autotrophs and $y$ is the number of grazers, and $h, i, j \in[1,2, \cdots, x], k, p, q \in[0,1, \cdots, y], x \in[1,2,3,4], y \in[0,1,2,3]$ and $x+y=4$.

The dynamics of these models are shown in Figures 3 and 4, demonstrating equilibrium dynamics in SC systems and pinball dynamics in PC systems. All species continuously coexist in the simulations until external extinction is imposed. This results in cascades of extinction in PC systems, where the extinction of one species triggers the extinction of other species, but not in SC systems. Such attributes (and others) can be predicted a priori, and even designed for. 

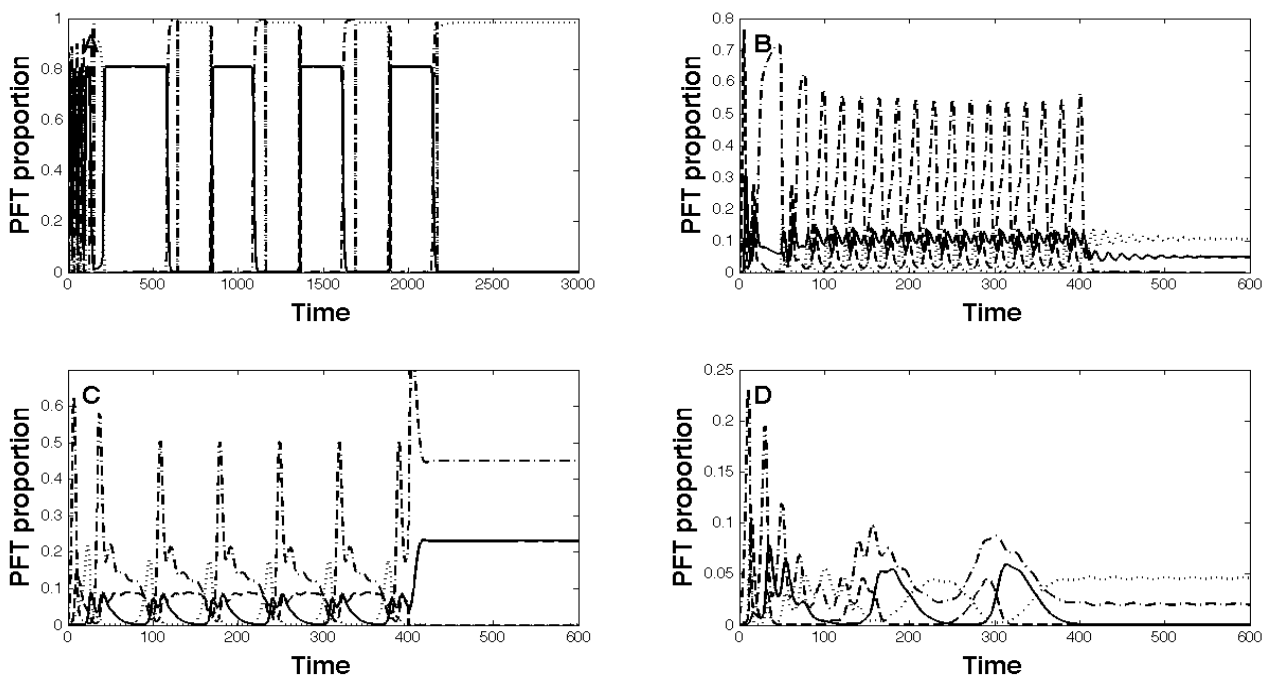

Figure 4. Time series for four example PC ecosystem models: four autotrophs (A); three autotrophs and grazer (B); two autotrophs and two grazers (C); and one autotroph and three grazers (D). These

PC systems were designed to have 'pinball' dynamics. Populations are shown as proportions of the total functional types (PFTs). Extinction is externally imposed on the third species (dashed line) at time $\mathbf{2 , 0 0 0}$ in (A) and time 400 in the other simulations. Predictable cascades of extinction occur as a result.

\section{Conclusions}

We define criteria for the construction of consistent ecologies in which the life functions that describe the growth, predation and mortality of each species, guild or functional type are constrained by generic principles. These three basic principles of independence of scale, conservation of mass and explicit resource limitation may be considered ecological axioms that ensure realistic ecologies. Our conditions provide an easy check for ecological consistency.

Our simple conditions allow us to formulate a new framework for the analysis of competition in ecosystem models. The framework provides a continuum of analytical solutions between very simplified theoretical models and very complex applied models. As a consequence, we have been able to describe the fundamental mechanisms that determine the outcomes of competition in a broad class of models. We have only considered dynamics of example models at the extremities of the spectrum of models that populate our framework, and are therefore unable to draw conclusions about any relationships between dynamics and coexistence.

\section{References}

Chesson, P., 2000. Mechanisms of Maintenance of Species Diversity. Annual Review of Ecology and Systematics 31, 343-366.

Cropp, R., Norbury, J., in press. The mechanisms of coexistence and competitive exclusion in complex plankton ecosystem models. Ecosystems.

Cropp, R.A., Norbury, J., 2009. Parameterizing plankton functional type models: insights from a dynamical systems perspective. Journal of Plankton Research 31, 939-963.

Gause, G.F., 1934. The Struggle for Existence. Williams and Wilkins, Baltimore.

Hardin, G., 1960. The competitive exclusion principle. Science (Washington D C) 131, 1292-1298.

Hastings, A., Powell, T., 1991. Chaos In A Three Species Food Chain. Ecology 72, 896-903.

Hutchinson, G.E., 1961. The paradox of the plankton. American Naturalist 95, 137-145.

Kishi, M.J., Kashiwai, M., Ware, D.M., et al, 2007. NEMURO_-a lower trophic level model for the North Pacific marine ecosystem. Ecological Modelling 202, 12-25.

Le Quéré , C., Harrison, S.P., Prentice, I.C., et al., 2005. Ecosystem dynamics based on plankton functional types for global ocean biogeochemistry models. Global Change Biology 11, 2016-2040.

Tilman, D., 2007. Interspecific competition and multispecies coexistence. In: May, R.M., McLean, A. (Eds.), Theoretical Ecology. Oxford University Press, Oxford, p. 257. 